CLÁUDIO MARCOS VIGNA

CAPACITAÇÃO DAS OPERAÇÕES INTERNAS PARA A CUSTOMIZAÇÃO EM MASSA: ESTUDOS DE CASOS NAS INDÚSTRIAS BRASILEIRAS

São Paulo

Universidade de São Paulo 
CLÁUDIO MARCOS VIGNA

\section{CAPACITAÇÃO DAS OPERAÇÕES INTERNAS PARA A CUSTOMIZAÇÃO EM MASSA: ESTUDOS DE CASOS NAS INDÚSTRIAS BRASILEIRAS}

Dissertação apresentada à Escola Politécnica da Universidade de São Paulo para obtenção do título de mestre em engenharia

São Paulo

Universidade de São Paulo

2007 
CLÁUDIO MARCOS VIGNA

\title{
CAPACITAÇÃO DAS OPERAÇÕES INTERNAS PARA A CUSTOMIZAÇÃO EM MASSA: ESTUDOS DE CASOS NAS INDÚSTRIAS BRASILEIRAS
}

\author{
Dissertação apresentada à Escola \\ Politécnica da Universidade de São Paulo \\ para obtenção do título de mestre em \\ engenharia \\ Área de concentração: Engenharia de \\ Produção
}

Orientador: Prof. Dr. Dario Ikuo Miyake

São Paulo

Universidade de São Paulo

2007 


\section{AGRADECIMETOS}

Ao Professor Doutor Dario Ikuo Miyake, pela sua paciência, dedicação e perseverança em me orientar. Ao CNPq pela Bolsa de Estudos. À família pelo auxílio e a todos os amigos que colaboraram direta e indiretamente, na elaboração deste trabalho. 


\section{RESUMO}

A Customização em Massa é uma estratégia de negócios relativamente recente que foi idealizada em meados dos anos oitenta, emergiu no meio empresarial em meados dos anos noventa, e, desde então tem sido adotada por muitas empresas devido ao diferencial competitivo que propicia. O objetivo desta estratégia é atender os desejos individuais dos clientes em grande escala e com maior lucratividade para a empresa. A adoção bem sucedida da Customização em Massa exige profundas alterações em áreas críticas das empresas. O objetivo do presente trabalho é, num primeiro momento, avaliar quais são essas áreas críticas que necessitam mudanças, e num segundo momento, quais são os elementos capacitadores necessários. O trabalho apresenta um modelo conceitual de definições sobre capacitação organizacional, competência funcional, técnicas operacionais e recursos organizacionais. Posteriormente verificou-se a influência destes elementos nos processos e como se dá a aplicação dos mesmos em diferentes setores industriais. A realização deste estudo baseou-se em cleta de dados por meio de questionários e na aplicação do método de estudo de caso em empresas de dois setores industriais, o automobilístico e o de alta tecnologia. A escolha destes setores justifica-se pelo fato deles se encontrarem, historicamente, na vanguarda da adoção das melhores práticas empresariais. Como resultado constatou-se que determinados elementos capacitam a empresa para a Customização em Massa em um setor mas não para outro, ou seja, não existe uma solução genérica para a capacitação organizacional em Customização em Massa. Observou-se também que a simples adoção dos elementos capacitadores não gera a competência funcional requerida em determinada área crítica para a geração desta capacitação organizacional; é também necessário que haja sinergia na aplicação destes elementos.

Palavras-Chave: Customização em Massa, Capacitação Organizacional, Indústria Automobilística e de Alta Tecnologia. 


\begin{abstract}
The customization is a recent strategy that appeared in the middle of 80's and currently it emerged in the enterprise environment. Such strategy has been adopted for many companies due to the competitive differential that it supplies. The objective of this strategy is to fulfill the most specific customers' desires in large-scale and with increasing profitability to the company. The successful adoption of Mass Customization demand deep changes in many critical areas of the enterprise.

The purpose of this paper is to analyses which are the critical areas that need to be changed, and which are the Mass Customization enablers. This research presents a conceptual definitions for resources, operational techniques, competences, and organizational capabilities required for Mass Customization. The paper presents a literature review on the issue of planning and deploying Mass Customization initiatives pointing out important resources and techniques that have been adopted by the adherents of this new strategy. It was perceived the influence of these enablers in its respective processes and its different applications in different industrial sectors. To this was adopted the method of case survey in two differents sectors, the automobile and high technology. This choice was proposital because this sector are in the vanguard of the best practices adoption. To have a valid classification of the enablers was elaborated two models. As a result was perceived that some enablers could be applied to one sector and not applied in others to reach the Mass Customization capacity. It does not exist a generic solution for a Mass Customization strategy adoption, because different sectors demands different enablers. It was also observed that the simple adoption of this enablers does not generate the enterprise competence demanded, being necessary the existence of a synergy between and focus of these enablers.
\end{abstract}

Key-words: Mass Customization, Enterprise Competence, Automobile and High Technology Industry. 


\section{LISTA DE ILUSTRAÇÕES}

Figura 2.1 - Evolução da CM a partir de outros paradigmas de produção

Figura 2.2 - Comparações entre as estratégias BTF e BTO............... 17

Figura 2.3 - Possíveis cenários......................................................... 20

Figura 2.4 - Nível de customização inteligente................................... 22

Figura 2.5 - Graus de customização do método de Pine...................... 23

Figura 2.6 - Produtos podem ser divididos em grupos baseados em pontos de customização.

Figura 2.7 - Capacitação organizacional sob a óptica de competências, técnicas e recursos.

Figura 2.8 - Capacitações fundamentais e pilares de gestão para a sustentação da CM.

Figura 2.9 - Relação entre tempo de setup e tempo de resposta.......... 44

Figura 2.10 - Agilidade de resposta e flexibilidade da mão-de-obra obtida pela evolução da sua autonomia.

Figura 2.11 - Posicionamento do DP conforme o tipo da estratégia de produção

Figura 3.1 - Classificação em relação ao grau de facilitação da Customização...

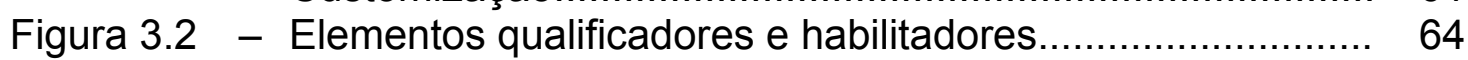

Figura 3.3 - Classificação dos elementos....................................... 64

Figura 4.1 - Princípios da CM e sua relação com competência e capacitação

Figura 4.2 - Ícones para construção de mapas de fluxo de valor........... 92

Figura 4.3 - Evolução das estratégias de operações na empresa estudada.

Figura 4.4 - Mapa do fluxo de valor da montadora de automóveis........ 95

Figura 4.5 - Operações de estampagem....................................... 96

Figura 4.6 - Operações de armação............................................ 97

Figura 4.7 - Operações de pintura.............................................. 98

Figura 4.8 - Setup do dispositivo de pintura.................................. 99

Figura 4.9 - Operações de montagem final..................................... 100

Figura 4.10 - Ilustração de uma linha de montagem final alimentada pela técnica JIS....................................................... 101

Figura 4.11 - Mapa do fluxo de valor da fabrica de computadores.......... 108

Figura 4.12 - Sistema de montagem de computadores........................... 110 


\section{LISTA DE QUADROS}

Quadro 2.1 - Diferenças entre as estratégias BTF e BTO....................... 14

Quadro 2.2 - Níveis genéricos de customização.................................... 28

Quadro 2.3 - Definições de recursos organizacionais............................. 31

Quadro 2.4 - Princípios da Customização em Massa.............................. 34

Quadro 2.5 - Codificação das referências bibliográficas.......................... 36

Quadro 3.1 - Questões de pesquisa................................................ 54

Quadro 3.2 - Exemplo de empresas internacionais que customizam seus produtos......................................................... 55

Quadro 3.3 - Elementos a serem analisados no estudo de caso............. 57

Quadro 3.4 - Elementos levantados na literatura x elementos a serem classificados............................ 58

Quadro 3.5 - Tabulação dos dados para obtenção da Classificação.......................................... 61

Quadro 3.6 - Exemplo de questionário: grau de facilitação da Customização...................................... 62

Quadro 3.7 - Classificação em elementos qualificadores e Habilitadores...................................... 67

Quadro 3.8 - Exemplo de questionário: para classificação de elemento em qualificadores ou habilitadores.... 67 


\section{LISTA DE TABELAS}

Tabela 2.1 - Competência Funcional em Operações Internas................. 42

Tabela 2.2 - Elementos capacitadores em Operações Internas.............. 43

Tabela 3.1 - Classificação do elemento setup rápido.............................. 62

Tabela 3.2 - Classificação do elemento ERP....................................... 63

Tabela 3.3 - Resultado da classificação do elemento setup rápido............................................ 68

Tabela 3.4 - Resultado da classificação do elemento Internet............................................ 68

Tabela 4.1 - Classificação obtida nas montadoras de automóveis....................................... 71

Tabela 4.2 - Classificação obtida nas montadoras de automóveis...................................... 76

Tabela 4.3 - Classificação obtida nos fabricantes de computadores................................... 80

Tabela 4.4 - Classificação obtida nos fabricantes de computador.................................... 84

Tabela 4.5 - Análise inter-setorial da classificação por tipo de influência....................................... 87

Tabela 4.6 - Análise inter-setorial da classificação em qualificadores e habilitadores.................... 89

Tabela 4.7 - Elementos com classificações divergentes como qualificador ou habilitador....................... 89

Tabela 4.8 - Elementos capacitadores para a CM e seu Impacto no processo produtivo de uma montadora de automóveis.

Tabela 4.9 - Elementos capacitadores para a CM e seu impacto no processo produtivo de um fabricante de computadores.

Tabela 4.10 - Elementos capacitadores para a CM identificados nos estudos de caso.

Tabela 4.11 - Elementos complementares identificados nos Estudos de caso................................. 117

Tabela A.1 - Competência Funcional em Planejamento de Produto e Processos.

Tabela A.2 - Competência Funcional em Logística de Abastecimento...

Tabela A.3 - Competência Funcional em Logística de Distribuição

Tabela A.4 - Competência Funcional em Marketing e Vendas.

Tabela C.1 - Perfil dos respondentes na amostra das montadoras de automóveis.

Tabela D.1 - Perfil dos respondentes na amostra dos fabricantes de computadores.

Tabela G.1 - Tabulação de dados para o setor automobilístico para a Classificação Conforme o Tipo de Influência. 
Tabela G.2 - Tabulação de dados para o setor automobilístico para a Classificação em Elementos Qualificadores e Habilitadores para a Customização

Tabela H.1 - Tabulação de dados para o setor de alta tecnologia para a Classificação Conforme o Tipo de Influência..........

Tabela H.2 - Tabulação de dados para o setor de alta tecnologia para a Classificação em Elementos Qualificadores

e Habilitadores para a Customização. 


\section{LISTA DE ABREVIATURAS E SIGLAS}

ABB

Asea Brown Boveri

AGM Auto gerenciamento da mão de obra

APM Arquitetura de produto modular

ATO Assemble to Order

B2C Business to Customer

BTF Build to Forecast

BTO Build to Order

CM Customização em Massa

CPU Central Processing Unit (Unidade de Processamento Central)

CRM Customer Relationship Management

DP Decoupling Point

ECR Efficient Consumer Response

EDI Electronic Data Interchange

ERP Enterprise Resources Planning (Software de gestão integrada)

ETO Engineering to Order

FPO Fluxo puxado conforme pedido

INT Internet

JIS Just in Sequence

JIT Just in Time

MDF Mão de obra multi-funcional

MRP Material Requirements Planning

MTO Make to Order

MTS Make to Stock

NASA National Aeronautics and Space Administration

PCP Planejamento e Controle da Produção

PCV Padronizado com variedade

PE Produção Enxuta

POS Postergação da diferenciação (postponement)

PSV Padronizado padrão sem variedade

RBV Resource Based View

RFM Robôs flexíveis no processo de manufatura

SET Setup rápido 
SO

Sistema Operacional

SRF

Sistemas de rastreamento de produtos

$\mathrm{TI}$

Tecnologias da Informação

VMI

Vendor Managed Inventory 